\title{
Phase field simulation of multiple phase transformation of Fe-C alloy
}

\section{during solidification process}

\author{
FENG Li ${ }^{1,2, a}$, JIA Beibei ${ }^{1, b}$, ZHU Changsheng ${ }^{1}$, LU Yang ${ }^{1}$, \\ XIAO Rongzhen ${ }^{1,2}$, FENG Xiaojing ${ }^{1}$ \\ ${ }^{1}$ Institute of Material Science and Engineering, Lanzhou University of Technology, Lanzhou, China. \\ 2 State Key Laboratory of Advanced Processing and Recycling of Non-ferrous Metals,Lanzhou, \\ China.

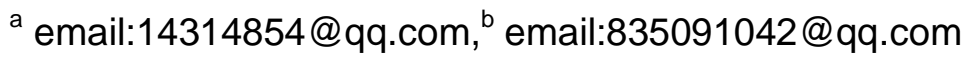

Keywords:phase field method, multiple phase transformation, Fe-C alloy,solute deposition Abstract:Coupling the phase field model of solid-solid phase transformation on the base of the liquid-solid phase transformation model,a new phase field model is established during solidification process.Taking the Fe-0.5mol-C alloy as an example, the model has realized the simulation of the liquid-solid-solid multiple phase transformation and has promoted the application of phase field simulation in Engineering.

\section{Introduction}

The phase field method has unique advantages in the simulation method of material microstructure ${ }^{[1]}$. Since there is no need to chase the interface, and it can also eliminate the restriction of long-range diffusion and long-range interaction which cannot be settled by MC method and CA method, the phase field method is rapidly becoming a very important and popular research method. It has been widely used in the field of materials, such as solidification ${ }^{[2]}$, grain growth $^{[3]}$, micro-segregation ${ }^{[4]}$, solid phase transformation ${ }^{[5-6]}$, dislocation motion and fracture ${ }^{[7]}$, Besides, its application range is still expanding. However, the process of phase field simulation is mostly concentrated on the liquid-solid phase transformation or solid-solid phase transformation alone, and the process of the real material solidification process often contains liquid-solid-solid multiple phase transformation, such as Fe-C alloy. Thus, in order to establish a quantitative relationship between the processing technology and the microstructure of the material, it is rather necessary to have liquid-solid-solid multiple phase transformation process. Therefore,the establishment of a liquid-solid-solid phase transformation model is of overreaching importance for the design of material composition and controlling the process of material shaping to obtain the required material properties.

This paper tries to establish a new model to simulate the process of multiple phase transformation via coupling the liquid-solid phase transformation model with solid-solid phase transformation process. Taking Fe-0.5mol-C as an example,we realized the simulation of multiple phase transformation during the solidification process, which has laid the foundation for the phase transformation and promoted the application of the phase field method in practical engineering. 


\section{model establishment}

phase field model

$$
\begin{aligned}
& \frac{\partial \phi_{1}}{\partial t}=M\left[\nabla \varepsilon \nabla \phi_{1}-f_{\phi_{1}}\right] \\
& \frac{\partial \phi_{2}}{\partial t}=M_{\phi}\left[K_{\phi} \nabla^{2} \phi_{2}+p^{\prime}\left(\phi_{2}\right) \bullet\left[f^{\gamma}\left(C^{\gamma}\right)-f^{\alpha}\left(C^{\alpha}\right)-\frac{\partial f^{\gamma}}{\partial C^{\gamma}}\left(C^{\gamma}-C^{\alpha}\right)+\lambda \bullet \Sigma X_{i}\left(\Delta T_{M}^{i}-\Delta T_{N M}^{i}\right)\right]-w \bullet g^{\prime}\left(\phi_{2}\right)\right] \\
& \phi_{1}+\phi_{2}=1
\end{aligned}
$$

The free energy density is introduced into the Fick diffusion equation for governing equations of the diffusion of carbon:

$$
\frac{\partial C}{\partial t}=\nabla\left[M_{C} \nabla\left(\frac{\delta F}{\delta C}\right)\right]=\nabla \cdot \frac{D(C, \phi)}{\partial^{2} f / \partial C^{2}} \nabla\left(\frac{\partial f}{\partial C}\right)
$$

In the formula (2), $\mathrm{D}(\mathrm{C}, \Phi)$ is the diffusion coefficient of carbon, and the expression is as below:

$$
D(C, \phi)=\left[D_{c}^{\alpha}\right]^{(P(\phi))} \cdot\left[D_{c}^{\gamma}\right]^{(1-P(\phi))}
$$

phase field parameters

The expression of $\mathrm{M}$ of the liquid - solid interface migration rate is as below:

$$
M^{-1}=\frac{\varepsilon_{0}^{3}}{\sigma \sqrt{2 W}}\left(\frac{1}{D} \zeta\left(c_{l}^{e}, c_{s}^{e}\right)\right)
$$

Among them, $\varepsilon_{0}$ and $W$ are phase field parameters, which are calculated by the interface and the interface thickness of lambda sigma, their expressions are as below:

$$
\begin{aligned}
& \varepsilon_{0}=\sqrt{\frac{6 \lambda \sigma}{2.2}} \quad, \quad W=\frac{6.6 \sigma}{\lambda} \\
& \zeta=\frac{R T}{V_{m}}\left(c_{l}^{e}-c_{s}^{e}\right)^{2} \times \int_{0}^{1} \frac{h(\phi)[1-h(\phi)]}{[1-h(\phi)] c_{l}^{e}\left(1-c_{l}^{e}\right)+h(\phi) c_{s}^{e}\left(1-c_{s}^{e}\right)} \bullet \frac{d \phi}{\phi(1-\phi)}
\end{aligned}
$$

The expression of $\mathrm{M}$ of the solid - solid interface migration rate is as below:

$$
M_{\phi}=K_{M} \cdot M_{p} \cdot \frac{2 \sigma}{K_{\phi}}
$$

$K_{M}$ is fitting coefficient, and $M p$ is the interface mobility of $\gamma-\alpha$ transformation in low carbon steel

$$
\begin{aligned}
& \sigma=\frac{\sqrt{K_{\phi} \omega}}{6 \sqrt{2}}, \quad 2 \lambda=\alpha \sqrt{2} \frac{\sqrt{K_{\phi}}}{\sqrt{\omega}} \\
& M p=0.035 \exp \left(-\frac{17700}{T}\right) m^{4} J^{-1} s^{-1}, K_{M}=-0.015 T+16.98
\end{aligned}
$$




\section{Numerical calculation}

\section{Material parameters}

Taking the research made by Toshio $^{[8]}$, M.Ode,Suzuki ${ }^{[9]}$ as reference object, in the paper,some material parameters have been introduced into the model, and the physical parameter of the Fe-0.5mol-C alloy are shown in the following table:

Table 1 physical parameters

\begin{tabular}{cc}
\hline Physical parameters & Fe-0.5mol-C \\
Grid cell size & $6.0 \times 10^{9} \mathrm{~m}$ \\
Melting point temperature Tm & $1810 \mathrm{~K}$ \\
Phase transformation temperature $\mathrm{T}_{0}$ & $1043 \mathrm{~K}$ \\
Interface energy $\sigma$ & $0.204 \mathrm{~J} / \mathrm{m}^{2}$ \\
Molar volume Vm & $7.7 \times 10^{-6} \mathrm{~m}^{3} / \mathrm{mol}$ \\
Gas constant R & $8.314 \mathrm{~J} /\left(\mathrm{mol}^{* \mathrm{~K})}\right.$ \\
\hline
\end{tabular}

\section{Initial conditions and boundary conditions}

In the coagulation process, the formation of the grain has spontaneous homogeneous nucleation, and non spontaneous heterogeneous nucleation. This new model in solidification process, the solid nucleation rate obeys Gauss distribution. At a certain degree of cold temperature, the number of solid core can be expressed as:

$$
n(\Delta T)=\frac{N_{\max }}{\sqrt{2 \pi} \Delta T_{\sigma}} \int_{0}^{\Delta T} \exp \left[\frac{\left(\Delta T-\Delta T_{\max }\right)^{2}}{2 \Delta T_{\sigma}}\right] d(\Delta T)
$$

In the model of liquid-solid-solid phase transformation, the number of nuclei in the heterogeneous nucleation is determined by the formula (10), according to the classical nucleation theory $^{[5]}$ :

$I=R \cdot N^{*}=R\left[N_{0} \cdot \exp \left(-\frac{\Delta G^{*}}{k_{B} T}\right)\right]=I_{o} \cdot \exp \left(-\frac{\Delta G^{*}}{k_{B} T}\right)$

\section{Numerical calculation}

This paper tries to use the explicit finite difference to solve the equations (1) and (2), which adopts the same time step $\Delta t$.and $\Delta t$ and space step $\Delta x$ (space step $\Delta x=\Delta y$ ) need to meet the following conditions of stability:

$\left.\Delta t<\min \left\{\Delta x^{2} /\left(4 D_{o}\right), \Delta y^{2} /\left(4 D_{o}\right)\right)\right\}$

Where $\mathrm{D}_{0}$ is the diffusion coefficient of austenite.

\section{Results and analysis}

The following figure shows the simulation results of liquid-solid-solid multiple phase transformation of the low carbon steel during solidification process, which simulated from our new phase field method.Fig. 1 is the simulation results of the liquid-solid phase transformation process.Fig.1 (a-c) show the progress of the grain grow up ,the grain morphology is from round ball after star shape become into the shape of dendrite with the passage of time goes under the action of anisotropy, and finally form a complex austenite dendrite. In the simulation region near the boundary of the grain boundaries, the dendrite branches are suppressed due to the boundary 
conditions. When the grains are not in contact with each other, every grain is allowed to free dendrite develop, with grain grow up, the growth of dendrite were subjected to bending or stop in the direction of the growth and the main crystal occurred inhibition under the of effect of grain, after inhibition the dendrite tip are deformed which is no longer a parabola in the theory of the Ivantsov ${ }^{[11]}$.Fig.1(d) shows that different colors represent different grains where the place which intersecting is the grain boundary,after the whole area become solid phase we don't find the boundary of dendrite arm in the same grain due to which no shown.

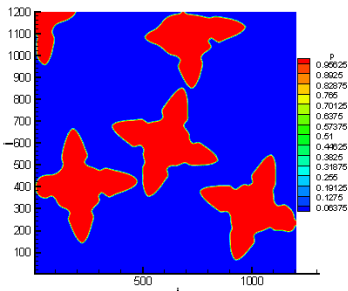

(a) $t=3000 \Delta t$

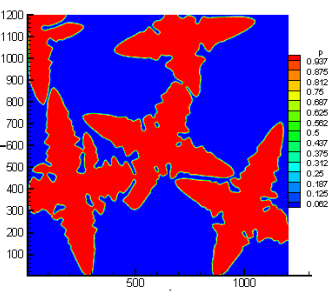

(b) $\mathrm{t}=5000 \Delta \mathrm{t}$

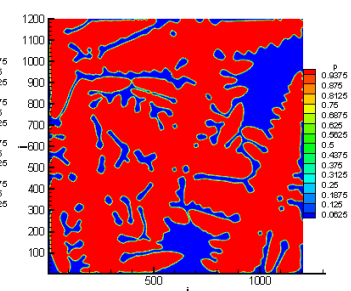

(c) $\mathrm{t}=8000 \Delta \mathrm{t}$

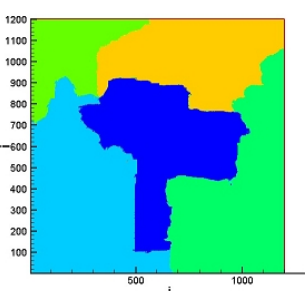

(d) $t=12000 \Delta t$

Fig 1. the simulation progress of austenite growth

With the decrease of temperature, the ferrite start to form core on the condition of austenite when ausenite transformed into ferrite, and grow up with the progress of nucleation.Fig.2(a) clearly shows that the priority nucleation of ferrite is on austenite grain boundary and some grain come into being in the internal defect of austenite, which nucleation consistent with the classical nucleation theory and the nucleation of rate is calculated by gaussian distribution. Fig.2 (a-d) show that the progress of the growth of ferrite grain,among Fig.2 (e) is the segregation of carbon correspond to Fig.2 (d), which show that the carbon atoms diffuse from the ferrite phase precipitate to the austenite phase which accumulation at the ferrite-austenite interface, and the carbon concentration is increased, which the concentration gradient of carbon atoms in the austenite is established.Under this concentration gradient, the transformation of ferrite by the carbon diffusion and crystal structure coarsening.Compared Fig. (1) with Fig.(2) we can seen that the rate of growth of austnite is faster more than the rate of ferrite due to diffuse rate of carbon in the progress of liquid-solid transformation is higher than in the progress of solid-solid transformation.

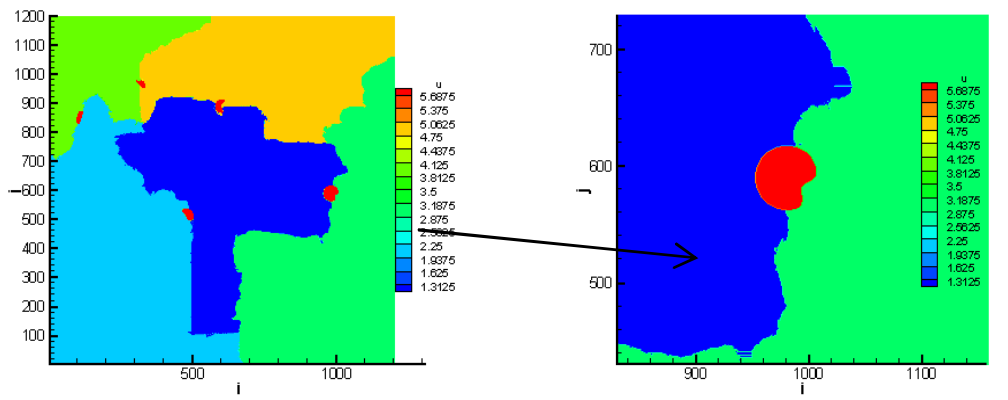

(a)The nucleation of ferrite in the austenite grain boundary (b)Enlargement of local area

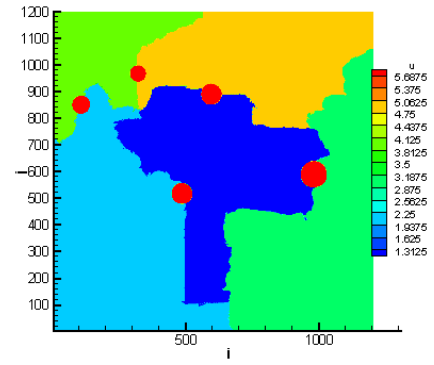

(c) $\mathrm{t}=4000 \Delta \mathrm{t}_{1}$

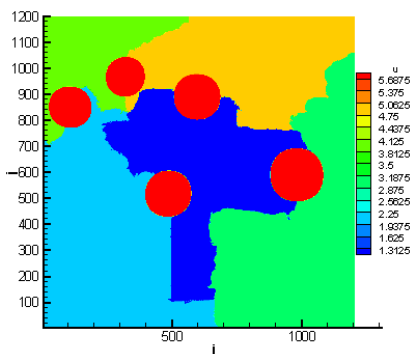

(d) $\mathrm{t}=9000 \Delta \mathrm{t}_{1}$

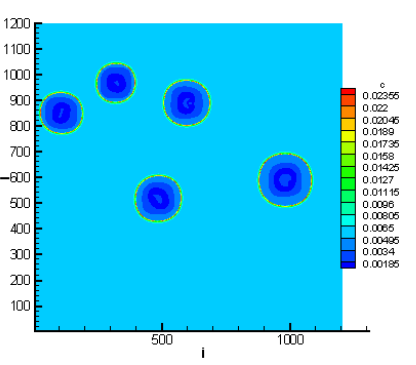

(e) solute distribution

Fig 2. (c-d) the simulation progress of ferrite growth,among $\Delta t_{1}$ is the time after nucleation (e) the solute distribution correspond to(d). 
The simulation results of the new model are compared with the results of $\mathrm{Niu}^{[10]}$, Loginova ${ }^{[9]}$, Huang ${ }^{[8]}$, Heo ${ }^{[5]}$ etc., which prove the results are in good agreement with the experimental results.Theremore based on the model and the application program have good physical mechanism and the crystal nucleus and growth is same with the transformation theory, which proves the feasibility of the new model.

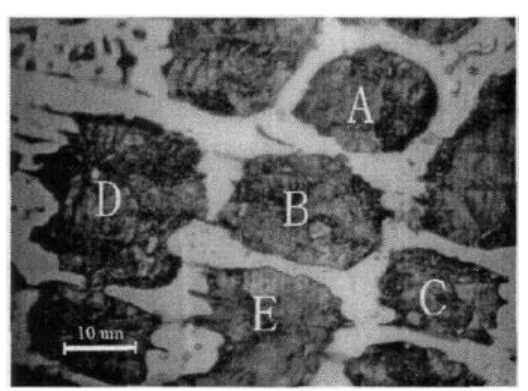

(a) Fe-C alloy morphology ${ }^{[10]}$

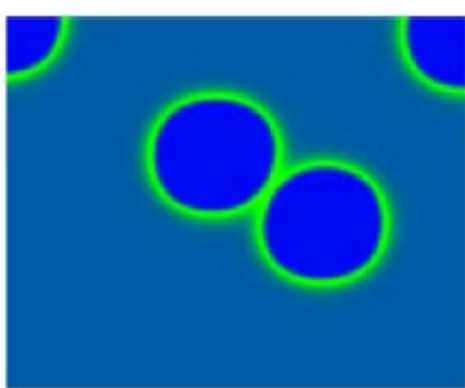

(b)the result is simulated by Huang ${ }^{[8]}$

Fig. 3 experiments and the results were made by predecessors

\section{Conclusion}

We briefly introduced the advantages of phase field method for simulation of multiple transformation,coupling liquid-solid phase transformation model with solid-solid phase transformation model which established a liquid-solid-solid multiple phase transformation model.In the case of Fe-0.5mol-C alloy, the simulation of the liquid-solid-solid multiple phase transformation by phase-field method has been realized.

The new model permits the observation of the future of ferrite nucleation,the results reveal that the diffuse regulation of carbon concentration, and the grain growth law of the austenite and ferrite are consistent with the classical transformation theory, which has established the foundation for the phase transformation process, as well as promoted the application of the phase field method in practical engineering.

\section{Acknowledgements}

This investigation is supported by the National Natural Science Foundation of China $(11504149,11364024)$.

\section{References:}

[1] Zhirong Luo .phase field modeling of microstructure evolution in metallic material[D].Doctoral Dissertation of Guangxi University. 2013.6.In Chinese

[2] B.Bottger·G.J.Schmitz·B.Santillana.Trans Indian Inst Met, 2012. 65(6):613-615.

[3] Li Feng,Changsheng Zhu,Phase-field simulation studies of dendrite growth coupling with force flow field[J].Advanced Materials Research Vols .2014.1069-1075.In Chinese

[4] Changsheng Zhu,Zhiping Wang,et al..Journal of Physics. 2006.55(3):1502-1507.In Chinese

[5] Tae Wook Heo,Long-qing Chen.Metals \& Materials Society, 2014. 66 (8):1520-1528.

[6] Ruixia Sun.Simulation for the austenite to ferrite transformation[D].Henan Polytechnic University.2010.6.In Chinese

[7] Xiu Ren, Jincheng Wang ,et al.Journal of Physics.2010.59(5):3595-3600.In Chinese

[8] Chen-Jiang Huang,David J.Browe,Shaun McFadden.Acta Materialia 2006(54):11-21.

[9] Loginoval,Odqvist J, Amberg G and Agren J. Acta Mater, 2003(51):1327-1339.

[10] Yane Niu,Phase-field simulation of Fe-C alloy in the isothermal solidification[D],Master 
Thesis of Xi'an Technological University.2008.5.In Chinese

[11] Li Feng,Zhi-Ping Wang et al.Journal of Physics,2008.57(02):1084-1090.In Chinese 